\title{
A REVIEW OF "EXISTENCE AND OPTIMALITY OF COMPETITIVE EQUILIBRIA" BY C. D. ALIPRANTIS, D. J. BROWN, AND O. BURKINSHAW*
}

David Ballard**

The theory of general economic equilibrium is surely one of the central achievements of modern economics. Although economists hold a wide range of views about the theory's usefulness as a guide to understanding or ameliorating the behavior of actual economies, its theorems and results concerning the efficiency of price-taking behavior in appropriately decentralized economic environments will no doubt continue to plague, perplex, or perhaps delight graduate students for years to come.

General equilibrium theory (GET) had its beginnings in the late nineteenth century in the work of L. Walras and F.Y. Edgeworth. It began to flower in the 1950's, with significant work done by K. Arrow, G. Debreu, and L. MacKenzie, among others. This body of work is perhaps most concisely articulated in Debreu's (1959) Theory of Value, which lays out theoretical results on the existence and efficiency of competitive equilibria in particular environments as well as a general framework for future research. In this framework, economies are represented by mathematical objects independently susceptible to rigorous formal mathematical analysis. Thus, the means of analysis and the interpretation of analytical results are conceptually distinct, which has the bcnefit of allowing researchers to express and communicate their results in an unambiguous and widely understandable language, that of mathematics. A related cost is that these research results can appear intimidating at best, and arbitrary and misleading at worst, to the mathematically unsophisticated.

\footnotetext{
*Aliprantis, C.D., Brown, D.J. \& Burkinshaw, O. 1990. Existence and Optimality of Competitive Equilibria. Berlin: Springer-Verlag, 284 pp., 38 figures. Softcover DM 58,- ISBN 3-540-52866-0.

**From The PhD program in Economics at the University of Illinois.
}

R. de Econometria Rio de Janeiro v. 12, no 2, pp.249-256 novembro 1992 
Nevertheless, this framework has guided much subsequent research in GET. Results from this research are primarily communicated to the economics profession through journal articles, but from time to time books and monographs bring together seemingly disparate research activities under a single cover. Such works are especial useful for students and for economists whose primary research interests lie outside of GET, since they serve to impose some order on otherwise scattered and possibly redundant research activities, and it is precisely such an order that the initiate might feel least qualified or able to create independently. A selective list of such works would include General Competitive Analysis (Arrow and Hahn, 1971), Core and Equilibria of Large Economies (Hildenbrand, 1974), The Theory of General Economic Equilibria, A Differentiable Approach (Mas-Colell, 1985), Foundations of the Theory of General Equilibrium (Balasko, 1986), and Equilibrium Analysis (Hildenbrand and Kirman, 1988).

The monograph under review, Existence and Optimality of Competitive Equilibria (henceforth EOCE), by C.D. Aliprantis, D.J. Brown, and O. Burkinshaw (henceforth ABB) is a recent and worthwhile addition to this list. The primary subject of EOCE is the status of competitive equilibria in economies having commodity choice spaces that are infinite dimensional. It organized a substantial body of research from the 1970's and 1980's into a systematic and sequentially organized whole. The representation of commodity choice spaces by infinite dimensional objects arises naturally when one considers commodities that differ in the time or place of their delivery, or when choice occurs under uncerlainly about which of many "states of the world" will obtain at the time of consumption. In this context, "infinity" can be either countable, with consumption choices represented by infinite sequences of real numbers, or uncountable, with consumption choices represented by functions on a continuum, such as $[0,1]$.

The monograph's first chapter presents the Arrow-Debreu theory of competitive equilibrium - defined as an allocation where, given prices, no consumer can achieve a higher level of satisfaction under her budget constraint, firms produce at feasible profit-maximizing 
levels, and all markets clear - when commodity spaces are finite dimensional. In such a setting, commodity spaces can be represented by $n$-dimensional Euclidean space, where $n$ is the finite number of commodities, from which a finite number of consumers choose subject to budget and feasibility constraints. While this chapter will likely be a review of known material for many readers, it serves to express familiar results in a language consistent with the new material introduced in the remainder of the book. This chapter could easily serve as an self-contained introduction to GET in Euclidean commodity spaces.

The remainder of the book generalizes this familiar theory to the infinite dimensional case. The numher of consumers and firms (producers) in the idealized economies is still finite, as in the ArrowDebreu theory, but these agents now face consumption and production choices defined over an infinite number of distinct commodities. If the nature of competitive equilibria could be obtained for such economies as simple limiting cases of larger and larger finite dimensional economies, there would be little need for independent research into infinite dimensional economies. Unfortunately, this is not the case. For technical (mathematical) reasons, many familiar results from the finite setting cannot be extended, in general, to the infinite dimensional setting.

Consider, for example, the positive orthant (or positive cone) of $n$-dimensional Euclidean space $\mathbf{R}^{n}$. This is the set $\mathbf{R}_{+}^{n} \equiv\{x \in$ $\left.\mathbf{R}^{n}: x_{i} \geq 0, i=1,2, \cdots, n\right\}$. It is easy to see that the interior of $\mathbf{R}_{+}^{n}$, int $\mathbf{R}_{+}^{n} \equiv\left\{x \in \mathbf{R}^{n}: x_{i}>0, i=1,2, \cdots, n\right\}$, is nonempty (that is, for any $x \in \operatorname{int} \mathbf{R}_{+}^{n}$, there exists some number $\delta>0$ such that the open ball $B_{\delta}(x) \equiv\left\{\mathbf{R}^{n}:|x-y|<\delta\right\}$ is wholly contained in int $\mathbf{R}_{+}^{n}$ ). However, the positive cone of an infinite dimensional space may have empty interior. The infinite dimensional space $C[0,1]$, the space of continuous functions on $[0,1]$, has as positive cone the set $C^{+}[0,1] \equiv\{x(t) \in C[0,1]: x(t) \geq 0,0 \geq t \geq 1\}$. If we use $\mathrm{d}(x, y) \equiv$ $\int_{0}^{1}|x(t)-y(t)| d t$ as a metric for the space, and let $\tau$ represent the topology on $C[0,1]$ generated by $\mathrm{d}$, it is easy to show that for any $x(t) \in C^{+}[0,1]$ that is strictly positive on $[0,1]$ and for any positive number $\delta$, there exists a function $y(t) \in C^{+}[0,1]$ such that $y(t)=0$ 
on some interval $(a, b) \subset[0,1]$ and $\mathbf{d}(x, y)<\delta$. This means that no open set in $\tau$ that contains $x(t)$ contains only functions that are strictly positive, so $C^{+}[0,1]$ has empty interior.

The inability to extend such seemingly basic topological results from the finite to the infinite dimensional setting affects the corresponding extension of general equilibrium results from the finite dimensional setting to the infinite dimensional one. This necessitates the independent analysis of competitive (price-taking) economies with infinite dimensional choice spaces. ${ }^{1}$ ABB note that "the fact that the economically interesting topologies on a commodity space $E$ give rise to an empty interior for $E^{+}$is the essential difference between the standard Arrow-Debreu model and the general equilibrium models which are the principal concern of this monograph" (EOCE, p.113).

The reader might correctly surmise from these problems that general equilibrium analysis in infinite dimensional settings requires a broader mathematical apparatus than its counterpart in finite dimensions. In chapter $2, \mathrm{ABB}$ present an overview of these new mathematical tools. This technical chapter is by no means selfcontained; the reader should have some prior knowledge of real and functional analysis and topology, especially the theory of dual topological vector spaces. Dual vector space structures arise because ABB, again following the analytic framework laid out by Debreu, represent prices by elements of a space dual to that of the underlying commodity space. $\mathrm{ABB}$ provide to the references to the relevant mathematics literature, but the works they cite are not at an introductory level. ${ }^{2}$

The authors extend general equilibrium analysis to the infinite dimensional setting by considering economies with commodity spaces that are Riesz spaces equipped with locally convex topologics. A Riesz space is a vector space $E$ that possesses a partial order - that is, a relation $\geq$ on $E \times E$ that is reflexive, antisymmetric, and transitive, similar to the "greater thall or equal to" relation on the reals - and has the property that if $x, y$ are in $E$, then $x \wedge y \equiv \sup \{x, y\}$

${ }^{1}$ For an excellent discussion of these problems see Larry Jones (1988). Most of the counter-examples presented by Jones show up at some point in EOCE.

2 An excellent introductory text in this context is Maddox (1988) 
and $x \Lambda y \equiv \inf \{x, y\}$ are also in $E$. For example, the space $C[0,1]$ is a Riesz space, but the space of everywhere differentiable functions $D[0,1]$ is not. (Consider the everywhere differentiable functions $x(t)=t$ and $y(t)=1-t, t \in[0,1]$. Although $x$ and $y$ are in $D[0,1]$, neither $x \wedge y$, which has a graph shaped like $\vee$, nor $x \Lambda y$, with a graph shaped like $\Lambda$, is differentiable at $t=1 / 2$, so neither is contained in $E$.) Local convexity of a topology $\tau$ on $E$, means that if $x \in E$ and $V_{x}$ is a neighborhood of $x$, then there is another neighborhood of $x, U_{x}$, that is convex and satisfies $U_{x} \subset V_{x}$. Intuitively, local convexity of a topology implies that the 0-element of $E$ can be regarded as a typical element in the sense that any neighhorhood $U_{x}$ of some $x \in E$ can be represented as a translation of a neighborhood $U_{0}$ of 0 by $U_{x}=U_{0}+x$. Since $n$-dimensional Euclidean spaces are examples of locally convex topological Riesz spaces, results from the general analysis of such spaces apply directly to the finite dimensional setting.

Chapters 3 and 4 of EOCE contain the extension of GET to exchange and production economies with locally convex topological Riesz commodity spaces. As the monograph's title indicates, the primary concern is the existence and (Pareto) optimality of competitive equilibria in such settings. Obtaining existence and the welfare theorems of GET requires that particular technical restrictions be placed on consumer preferences and production technologies. These restrictions, introduced by T. Bewley and A. Mas-Colell, are necessary for overcoming the empty interiors that characterize the positive cones of many infinite dimensional Riesz spaces. It is impossible to be more explicit about these restrictions on agent characteristics without directly reproducing large portions of $\mathrm{EOCE}$, but the presentation of these issues in EOCE is thorough and clear.

The final chapter applies the analytic techniques previously developed to Overlapping Generation (OLG) models, which ABB describe as "the other major paradigm in general equilibrium theory." OLG models introduce a dynamic element that the Arrow-Dehreu framework conspicuously lacks, and have been extensively used by theorists in dynamic microeconomics and macroeconomics. I assume that readers are familiar with the general setup of OLG exchange models with infinite time horizons and finite lived generations of con- 
sumcrs. An important characteristic of many OLG models is the existence of competitive equilibria that are Pareto inefficient (suboptimal), which contradicts the First Welfare Theorem of standard Arrow-Debreu theory ${ }^{3}$ ABB analyze OLG models in which each generation may have preferenees over an infinite dimensional commodity space, which are of course represented by loeally convex topologieal Riesz spaces, with prices represented by spaces dual to the commodity space. In this setting, which is much richer mathematically than these brief remarks can convey, ABB establish conditions that ensure the existence of effieient competitive equilibria for OLG economies.

EOCE' contains an ample supply of examples and illustrations that help to anchor the reader's intuition and comprehension. The numerous exercises that follow each chapter section are an especially welcome feature of the book: "learning by doing" is a principle that applies to more than endogenous growth theory.

It is useful to compare briefly EOCE with another recent text, Hildenbrand and Kirman's Equilibrium Analysis (EA). ${ }^{4}$ As readers of EA will recall, Hildelbrand and Kirman emphasize the interesting relationship that exists between competitive, or Walrasian, equilibria - a concept based on noncooperative price-taking behavior in completely decentralized economic environments - and the core of an economy - a concept rooted in cooperative behavior within coalitions of agents in an exehange economy. Under appropriate circumstances, core allocations - ones that are individually rational for all agents and Pareto eficient - exist in an economy, and the core allocations that can be supported by prices are competitive equilibria as well. In the years since 1976, when the initial version of EA was published, the syllogism a) under certain circunstances, core allocations exist and b) some or all of these core allocations can be supported by prices imply c) competitive (quasi-) equilibria exist as well has become part of the common toolkit of mathematical economics. ABB therefore use, with little or no fanfare, techniques and relationships

\footnotetext{
${ }^{3}$ The First Welfare Theorem states that when preferences are convex, competitive equilibria are Pareto efficient. This is usually due to problems inhibiting intergenerational trade. For an example of its violation in OLG models see Paul Samuelson's seminal paper (1958).

${ }^{4}$ Hildenbrand and Kirman (1988).
} 
that Hildenbrand and Kirman chose to emphasize. Such differences in emphasis reflect differences in the authors' tastes as well as the passage of time, and because of this, a reader of EOCE who is unfamiliar with EA will profit by becoming acquainted with it as well.

With EOCE, Aliprantis, Brown, and Burkinshaw have provided graduate students and researchers in economics and mathematics with a thorough and readable course covering significant aspects of modern general equilibrium theory. They have managed to combine gracefully technical rigor with intuitive examples and exercises, and the reader who perseveres through its pages will find herself amply rewarded.

\section{Referências}

Arrow, K. \& Hahn, F. 1971. General Competitive Analysis. San Francisco: Holden Day.

Balasko, Y. 1986. "Foundations of the theory of general equilibrium." Working paper 86-22, Center of Analytic Research in Economics and the Social Sciences, University of Pennsylvania.

Debreu, G. 1959. Theory of Value. Cowles Foundation Monograph 17. New Haven: Yale University Press.

Hildenbrand, W. 1974. Core and Equilibria of a Large Economy. Princeton: Princeton University Press.

Hildenbrand, W. \& Kirman, P. 1988. Equilibrium Analysis: Variations on Themes by Edgeworth and Walras. Amsterdam: NorthHolland.

Jones, L. 1986. "Special problems arising in the study of economies with infinitely many commodities." In Sonnenschein, H., ed., Models of Economics Dynamics. Spring-Verlag.

Maddox, I. J. 1988. Elements of Functional Analysis. Cambridge: Cambridge University Press.

Mas-Colell, A. 1985. The Theory of General Equilibrium - A Differentiable Approach. Cambridge: Cambridge University Press. 
Resenha bibliográfica

Samuelson, P. 1958. "An exact consumption-loan model of interest with or without the social contrivance of money." Journal of Political Economy 66:467-482. 